\title{
Research on the Application of Corrosion On-line Monitoring System in Oil Refining Industry
}

\author{
Pan Yi, Zhang Liming, Huang Chong and Yang Shuangchun \\ (Liaoning Shihua University, Fushun, Liaoning, China 113001)
}

\begin{abstract}
Corrosion monitoring has increasingly become a focused major issue of oil refining industry. As is well-known, there are a great deal of impurities causing serious corrosion to pipeline, reducing working life of petroleum pipeline. In recent years, our country refines high-sulfur crude oil, equipment corrosion problems become more prominent.Under the background of anticorrosive in science, this paper introduces some common using monitoring technologies, latest corrosion monitoring system, and discusses details the system structure and working principle. This system can collect corrosion data from oil pipeline synthetically and effectively, analyses scientifically corrosion data, understands the extent of the corrosion, studies corrosion rule, predicts corrosion trend, finds the reason of corrosion, puts forward the anticorrosion scheme, which provides decisionmaking basis for corrosion protection scientifically.
\end{abstract}

Keywords: - corrosion, pipeline, monitoring system

\section{INTRODUCTION}

Overseas, many experts of petroleum chemical industry have developed a lot of issues fundamental research about pipeline corrosion, established a better monitoring method, and set up with suitable management. As the most developed country in the world, America attaches great importance to the oil pipeline corrosion monitoring and protective work, and does a lot of productive research in the field, developed many corrosion monitoring products. Because these products have reliable performance, high accuracy of monitoring, they have a wide range of applications in petrochemical refining field.

At home, the rapid develop economy promotes the development of corrosion monitoring theory and technology, although started later, the development speed is fast. Many research institutes and enterprise are joined into corrosion monitoring field. Learning advanced technologies from abroad, developing a lot of corrosion monitoring equipments, which makes tremendous contributions to the development of domestic corrosion monitoring career.

With the development of technology, corrosion monitoring begins to appear the trend of the real-time online change, wireless, intelligence, and the network[1]. Especially, zkwell recently introduces an independent research of new products "online corrosion wall thickness monitoring system", which changes the model of the traditional ultrasonic thickness by human, realizes real-time collection of wall thickness measurement, expels from the human interference factors, and be used in high temperature dangerous area or other remote area, application prospect is very good.

\section{Domestic Commonly Used Several Corrosion Monitoring Method And Application}

\section{Current Situation}

At present, domestic corrosion monitoring methods mainly contains monitoring hole method, weightlessness hanging piece method, resistance probe, electrochemical, inductive method etc, in industrial applications, these theories and technologies is also constantly applied and developed[2] .

\subsection{WEIGHTLESSNESS HANGING PIECE MONITORING METHOD}

Weightlessness hanging piece method is a classic monitoring corrosion rate method. Through the regular take-put specimens method, testing or evaluating pipe or container of corrosive medium, and through the known surface area, the initial quality, the reducing quality due to corrosion, corrosion time, calculating corrosion rate, and then judge the pipe or container average service life. This method is the most direct, efficient and economic corrosion assessment method, but monitoring cycle is long, and the human interference factors is existent.

\subsection{RESISTANCE PROBE MONITORING METHOD}

Resistance probe monitoring method is through the metal components (resistance probe) cross sectional area reducing because of corrosion, and thereby, which makes resistance increasing. If metal corrosion in general is uniform, so the resistance change rate is direct ratio with the metal corrosion volume. CST410 resistance probe monitoring instrument uses the reversing current method, effectively compensating the 
influence of heat motor-driven power and contact motor-driven power, at the same time, integrating temperature sensor in resistance probe, and through the nonlinear automatic compensation technology, improving the actual environment monitoring accuracy in the resistance probe temperature fluctuations, and at the same time, improving the technology reliability in the acidic gas fields application environment. The foreign matter existing in the environment media temperature, flow velocity, metal material and surface of probe (such as corrosion products), they will affect the accuracy of the resistance probe measurement results [3].

\subsection{HYDROGEN PROBE MONITORING METHOD}

In the acidic medium, producing the hydrogen in metal surface due to corrosion, they are ion or atom state to spread into internal metal, So that the metal produce the trend of hydrogen drum bubble, crisp and hydrogen-induced cracking. Because hydrogen probe measurement results can't directly calculate corrosion rate, therefore need to combine with other corrosion monitoring method. CST820 hydrogen corrosion test unit bases on constant flux potential transient polarization mode, uses the potential square wave incentive model, through the current-time attenuation curve-fitting the atoms of hydrogen concentration measurement. This unit mainly includes data acquisition part, automatic measurement control section, data storage part, and data output part.

\subsection{ELECTROCHEMICAL NOISE MONITORING METHOD}

Electrochemical noise is to point to be under the constant potential (or constant current) control, in electrolysis pool, the spontaneous fluctuations of metal electrodes/solution current interface (or electrode potential). Electrochemical noise measurement is based on random process theory as a foundation, study corrosion process electrode/solution potential and current wave regularity with the statistical method, which is a novel electrochemical research methods. In the electrochemical power system evolution process, the system electrical state parameter happens to the random fluctuation balance phenomenon with time, this provides a rich of evolution information, including system information from quantitative change to the qualitative change. CST530 electrochemical corrosion monitoring instrument analyses the area of the noise peak, strength, rising and falling rate, and the frequencies of occurrence, gets steady or sub-steady erosion point, crack development ,stress corrosion, and many other development information of local corrosion.

\subsection{INDUCTANCE PROBE METHOD}

Inductance probe method synthesizes characteristics of the electrochemical probes fast response and the wide range use of resistance probe, response time is faster 2 to 3 orders of magnitude than resistance probe, and applicable medium conditions more widen than electrochemical probes. At the same time, with the high sensitivity, strong anti-interference, in low temperature, the inductance probe can take the place of the resistance probe.

\section{REAL-TIME ONLINE ANTI-CORROSION MONITORING SYSTEM STRUCTURE AND WORKING PRINCIPLE}

For better anticorrosive work, reduce labor intensity and guarantee the safety of workers, avoid artificial ultrasonic monitoring, and choose inductance probe, ultrasonic sensor real-time online monitoring method, this system operating costs is lower, reducing artificial factors, better effect of anticorrosive, more accurate than the artificial thickness, which is another new choose for refining enterprise [4].

\subsection{CHOOSE THE SUITABLE THICKNESS MONITORING STATIONS}

The basic principle of determining the measuring thickness point: set measuring thickness points in straight and bent pipe section, while increase thickness points in the danger point, increase measuring density in severely corrosive place (high temperature, low temperature sulfur corrosion parts wet $\mathrm{h} 2 \mathrm{~s}$ corrosion parts, the naphthenic acid corrosion parts etc). Standard the probe stationing position, strengthen the rationality of the probe monitoring: on the one hand, choose the representative position to monitor equipment pipeline corrosion situation, on the other hand, easy to overhaul and vindicate probe [5].

\subsection{CORROSION MONITORING TECHNOLOGY SELECTION}

Electrochemical method can quickly get data (5 to 15 minutes), directly measure the corrosion rate, and thus get a wide range of application[6]. But it does not apply to the nonelectric conduction medium, also does not apply to multiphase or discontinuous medium, the same does not apply to contain adhesion material (such as oil dirt etc, which will cover electrode). And inductance method has increasingly become the main monitoring technology of refining enterprise using, here use inductance method as the example, this paper introduces the construction process of anticorrosion online real-time system. 


\subsection{CONSTRUCTION OF REAL-TIME ONLINE ANTICORROSIVE MONITORING SYSTEM}

According to the principle of the inductance probe monitoring, develop the on-line inductance probe monitoring technology[7], this technology mainly include: the corrosion data collector, corrosion inductance probe and data remote transmission system, this system is mainly inductance probe installed in process piping, through the corrosion data collector, acquiring the original analog signals relative with the corrosion rate, amplification converted into digital signals, and the transmission to monitoring room computer. RS-485 [8] is connected data collector and computer interface, it makes the data transmission distance to 1200 meters, data at the computer show and the establishment of database post-processing, through the multicast real-time on-line monitoring, get the corrosion situation of the production process, which will integrate various probe monitoring data, monitoring data can reach device control room of the same computer terminal, and rely on internal local area network and Internet platform, establish the online monitoring system network covering plant with set data storage retrieval alarm as one, through the Internet, support to realize corrosion data of different management, make each department master device corrosion information quickly, reach information resources share, which has important practical significance to anticorrosion work. To prevent accident and reasonably arrange regular maintenance, must also measure thickness at designated time and place. All kinds of offline detection accumulated data and online monitoring data constitute the corrosion test database, and thereby establish the corrosion monitoring and detection nets [9], the system structure is drawing in the figure 1.

\subsection{Measures selection based on the real-time anticorrosion monitoring feedback information}

Through optimizing crude oil processing scheme, strengthen the constant reduced pressure "a take off two note"[10] operation, vacuum residua fractions and catalytic slurry oil systems use scale inhibitor, reduced pressure systems use high temperature corrosion inhibitors etc, we can effectively reduce equipment corrosion [11], and extend the device operating cycle. According to the wall thickness measuring point corrosion rate, combined with results of bypass kettle hanging piece, the tower hanging piece, and other monitoring methods, provide the basis for the key parts of the corrosion materials selection. System and realize the real-time data acquisition, the user can understand promptly equipment corrosion situation. And realize the historical data statistic and analysis, provide the basis for users to improve production craft. According to the feedback data to adjust the production craft, such as adjusting water tower, note ammonia, note the cycle of corrosion inhibitors or injection quantity, choose the best technology of slow equipment corrosion.

\subsection{A NEW ANTICORROSION MONITORING SYSTEM}

Because the ultrasonic method to determine the artificial collection of wall thickness data is often repetitive bad and data records error, London Imperial University nondestructive testing team has developed a new type of monitoring system [12], the working principle: installed in the wave of stainless steel ultrasonic sensor welding in two bolts fixed in the pipeline and container, it is the core of the continuous monitoring system, the wave pipe isolates electronic equipments and sensors with extreme temperature, and make the ultrasonic signal to wall, and back to the ultrasonic signal, but not make the signal too much attenuation and distortion [13]. Through frequently measuring wall thickness, we can acquire metal loss situation, the measurement precision can reach dozens of microns. Each sensor is equipped with radio equipment, and communicated with the other sensors and nets between connector (base station) within 50 meters (55 yards) [14]. Many of the sensors constitute a wireless network that does not need to install the previous wireless network infrastructure. Radio equipment of each sensor has the role of the transmission or repeat send, which can make the network expand to hundreds of meters area from nets connector. Through the base station, the data can be sent to the computer database connected with it. If the computer is already networking, then based on the browser's visual software, the checking corrosion engineers can see these data on their work stage [15].According to different process monitoring apply situations, these data can be output at the request of different file formats, thereby realize the seamless transmission and read to the related packets, at the same time, and be related with craft data in parts of the sensor located. The system monitoring of pipe wall thickness range change is $3 \sim 40 \mathrm{~mm}(1 / 8 \sim 11 / 2$ inch), which can be used for a wide range of steel and other material alloy occasions [16]. The sensor is supplied by the battery power. Thus do not need to lay cable, which can make the installation cost reach to the minimum, but also few restrictions for remote areas and large-scale use.

\section{CONCLUSIONS AND EVALUATION}

With the composition of crude oil becoming more bad, impurity composition go up constantly, put forward higher request on production process, and corrosion problems emerge in with it[17], corrosion monitoring system is the inevitable requirement of new type oil refining industry.

Online monitoring can provide the scientific basis for process protection measures implementation, helpful to perform effective anti-corrosion process adjustment, control corrosion occurred[18]. Online monitoring can provide timely and accurate monitoring data while crude oil properties changing, which is 
beneficial to the analysis of corrosion accumulation data, according to the monitoring data, predict the service life of equipment, provide the scientific basis for enterprise's long-term corrosion monitoring and warning, and key device equipment materials upgrading[19]. Strengthen the inductance probe maintenance management, if probe works abnormally, use the simulation probe supplied to checkout and confirm further, if the simulation probe testing results still shows the fault, you need to contact with the manufacturer treatment as soon as possible. Strengthen fixed-point thickness, the proposal is measuring thickness near the monitoring nod, thickness point site should have obvious mark, have a unified and standard Numbers, guarantee the accuracy of the numerical monitoring, provide reference data for the online monitoring.

\section{Acknowledge}

This research work is supported by Doctor Fund of Liaoning Shihua University (No. 2012XJJ-011).

\section{REFERENCE}

[1] Yong Zeng. Comparative online monitoring system in the application of distillation unit[J].Journal of Petroleum Chemical Corrosion and Protection, 2011, 3:47-50.

[2] Xikui Gu, Tan Gu, Jing Li , Yong Huang, Yue Wang. A variety of corrosion monitoring system in the application of the peak stood[J].Journal of Petroleum and Natural Gas Chemical Industry,2011,3.

[3] Liqun Zheng, Zegui Wan, Nan Gao, Chengfeng Zhang. Refining corrosion monitoring technology application and device progress[J].Journal of Petroleum Chemical Corrosion and Protection, 2012, 2:47-50.

[4] Zhenglai Liu. Corrosion of online monitoring system for the application in common pressure-relief device [J]. Chinese Science and Technology Information, 2007 (7):69-71.

[5] Zhihua Yu, Xianggang Meng. Corrosion monitoring technology and its application in oil and gas field[J]. Pipeline Technology and Equipment, 2012, 2:48-49.

[6] Peter Collins. Consider to use a new type monitoring system to prevent corrosion[J]. Hydrocarbon Processing, 2012, 3:20-23.

[7] Jinyang Zhang, Jugang Luo, Hongsheng Tai. The corrosion behavior of reinforced concrete and steel corrosion monitoring technology[J].The People Yellow River, 2011, 4:112-116.

[8] Xiaohui Yang, Jiyang Rao, Yannan Wang. Application of online monitoring technology in petrochemical industry[J].Journal of Petroleum Chemical Corrosion and Protection, 2011, 3:40-42.

[9] Faping Yang, Guibo Wang. Puguang gas field ground airtight gathering and transportation system corrosion monitoring and control system[J]. Natural Gas Industry, 2012, 1:94-97.

[10] Qiang Zhang, Wen Chen, Mengwei Yang, Maoqiu Liu, Shuang Peng, Tan Gu. High acidic gas field corrosion monitoring technology research[J]. Oil and Natural Gas Chemical Industry, 2012,1:62-65.

[11] Qiang Zhang, Yongfan Tang, Tan Gu. ChuanYu gas purification plant desulfurizing devices monitoring technology research and application[J]. Oil and Natural Gas Chemical Industry, 2010, 2:548-551.

[12] Guoxiang Zhao. A new type corrosion monitoring device in the application of water injection system [J].Journal of Petroleum Chemical Industry Equipment, 2011, 3:72-74.

[13] Ying Yang, Kai Wei, Lin Zhang, yi Yang. High sulfur content corrosion monitoring technology application gas field study[J].Corrosion Science and protection technology, 2011 5:449-450.

[14] Zhiping Zhang,Junbi Liao, Zhengjun Wan, Chuanhe Xiao. Pipeline corrosion monitoring system with a pit corrosion monitoring ability [J]. Electronic Measurement Technology, 2012, 2:92-96.

[15] Sérgio Reis Cunha, Renata Gonçalves, Sérgio Rui Silva and Ana Dulce Correia. An automated marine biomonitoring system for assessing water quality in real-time[J]. Ecotoxicology, 2008, 17( 6):558-564.

[16] A. Buyuksagis, S. Erol. The Examination of Afyonkarahisar's Geothermal System Corrosion[J]. Journal of Materials Engineering and Performance, 2012,6.

[17] G. Quintana, M. L. Garcia-Romeu and J. Ciurana. Surface roughness monitoring application based on artificial neural networks for ball-end milling operations[J]. Journal of Intelligent Manufacturing, 2011, 22(4):607-617.

[18] Balakrishnan Anandkumar and Wenxin Shi. Corrosion: monitoring and control[J]. Reviews in Environmental Science and Biotechnology, 2009, 8( 4): 317-319.

[19] [Andrea G. Capodaglio and Arianna Callegari .Online Monitoring Technologies For Drinking Water Systems Security[J]. NATO Science for Peace and Security Series C: Environmental Security, 2009, Risk Management of Water Supply and Sanitation Systems, 3:153-179. 